\title{
Acute Operative Management of Osteoporotic Vertebral Compression Fractures Is Associated with Decreased Morbidity
}

\author{
Emily S. Mills, Andy T. Ton, Gabriel Bouz, Ram K. Alluri, Raymond J. Hah \\ Department of Orthopaedic Surgery, Keck School of Medicine, University of Southern California, Los Angeles, CA, USA
}

Study Design: Retrospective national database study design.

Purpose: This study was designed to determine whether acute percutaneous vertebral augmentation (PVA) alters morbidity compared with nonoperative management.

Overview of Literature: Osteoporotic vertebral compression fractures (OCFs) are common and represent a large economic and patient burden. Several recent studies have focused on whether PVA offers benefits compared with nonoperative treatment.

Methods: A retrospective cohort analysis was conducted using the Nationwide Inpatient Sample from 2015 to 2018. Patients with nonelective admissions for OCFs were identified using International Classification of Diseases (10th edition) codes. The exclusion criteria included age of less than 50 years, fusion and decompression procedures, and the presence of neoplasms and infections. Propensity score matching was implemented to construct 2:1 matched cohorts with similar comorbidities at admission. The patients were divided into the operative and nonoperative treatment groups. Univariate and multivariate regression analyses were performed to compare differences in in-hospital complication rates between the groups. All $p$-values of less than 0.05 were considered significant.

Results: We identified 14,850 patients in the operative group and 29,700 patients in the nonoperative group. In the multivariate analysis, operative treatment was associated with significantly lower rates of pneumonia (odds ratio [OR], $0.75 ; p<0.001$ ), acute respiratory failure (OR, $0.84 ; p=0.009)$, myocardial infarction $(0 \mathrm{R}, 0.20 ; p<0.001)$, acute heart failure $(0 \mathrm{R}, 0.80 ; p=0.001)$, cardiogenic shock (OR, 0.23; $p=0.001$ ), sepsis (OR, 0.39; $p<0.001$ ), septic shock (OR 0.50; $p<0.001)$, and pressure ulcerations $(0 \mathrm{R}, 0.71 ; p<0.001)$. However, operative treatment was associated with a significantly greater risk of acute renal failure (OR, 1.19; $p<0.001)$ than nonoperative treatment.

Conclusions: Patients who undergo acute PVA for OCFs have lower rates of respiratory complications, cardiac complications, sepsis, and pressure ulcerations while having a higher risk of acute renal failure.

Keywords: Osteoporosis; Compression fracture; Vertebroplasty; Kyphoplasty

Received Jul 22, 2021; Revised Aug 29, 2021; Accepted Sep 28, 2021

Corresponding author: Emily S. Mills

Department of Orthopaedic Surgery, Keck School of Medicine, University of Southern California, 1200 N. State St., GH 3900, Los Angeles, CA 90033, USA

Tel: +1-303-905-1123, Fax: +1-323-442-5301, E-mail: Emily.mills@med.usc.edu 


\section{Introduction}

Osteoporotic compression fractures (OCFs) are the most common osteoporotic fracture among the aging population, comprising $27 \%$ of all osteoporotic fractures [1]. They represent a large economic burden on the healthcare system. In 2015, OCFs had an incidence of 102.1 per 10,000 Medicare beneficiaries, costing approximately $\$ 658$ million [2]. Of these patients, 35\% required acute hospitalization within 7 days following OCF, and $61 \%$ required hospitalization greater than 8 days after OCF. Furthermore, the 1-year mortality rate following OCF among Medicare beneficiaries was $21 \%$.

Recent literature has focused on treating OCFs. Operative management consists of percutaneous vertebral augmentation (PVA), including vertebroplasty and kyphoplasty, whereas nonoperative management consists of pain management and external orthoses [3]. Several randomized controlled trials did not show a significant clinical benefit regarding pain or functional outcomes with vertebroplasty [4-7]. In contrast, several studies have found that in the aged population, vertebroplasty offers improved functional outcomes and morbidity and mortality benefits $[8,9]$. It is not currently recommended in national clinical guidelines for treating OCFs.

Several studies have been conducted to elucidate the benefits of kyphoplasty versus nonoperative treatment for OCFs. Numerous studies have shown that kyphoplasty offers greater improvement in daily activities, quality of life, and vertebral deformity than conservative treatment $[10,11]$. Patients treated operatively have also been found to have improved mortality benefit [12]. Currently, clinical practice guidelines weakly recommend kyphoplasty [13].

However, the number of studies examining and comparing acute complication rates between operative and nonoperative management of these fractures in patients hospitalized for OCFs is limited. This study was designed to determine whether acute PVA alters morbidity compared with nonoperative management.

\section{Materials and Methods}

\section{Data source}

The Nationwide Inpatient Sample (NIS) is a national database within the Healthcare Cost and Utilization Project published annually and provides nationally representative information on over 7 million hospitalizations across the United States. The NIS is the only national database that uses discharge weights, allowing accurate estimations of nationwide incidence of diseases and procedures. Additionally, the NIS captures all patients, regardless of insurance status. The database uses International Classification of Diseases, 10th revision (ICD-10) codes for documenting all diagnoses and indicated procedures per hospital admission. Given the nonspecific nature of ICD-9 coding used in years before 2015, this study only analyzed NIS years 2015-2018 to use the additional granularity offered by ICD-10 coding. This study did not require Institutional Review Board approval as all patient data within the NIS is deidentified.

\section{Patient selection}

Patients who were nonelectively admitted for OCFs from the 2015 to 2018 NIS dataset were identified using ICD10 codes. The patients were then divided into those who underwent operative treatment and those who received nonoperative treatment. Operative treatment was defined by the use of PVA, by either vertebroplasty or kyphoplasty. Patients aged less than 50 years, those who were surgically indicated for fusion and decompression procedures, and those with neoplasms and infections were excluded from the study. ICD-10 codes used in defining the diagnosis of osteoporotic compression fracture, the operative treatment group, and exclusion criteria are specified in Table 1.

\section{Statistical analysis}

Nearest-neighbor propensity score matching 2:1 cohorts for age, total comorbidities, year of procedure, calculated risk of mortality at admission, and calculated illness severity at admission was performed. Univariate analysis was used to assess differences in demographic characteristics, costs, length of stay (LOS), and complication rates between the operative and nonoperative groups. Multivariate logistic regression controlling for significant comorbidities and demographic characteristics was performed to analyze the relationship between either operative or nonoperative OCF treatment and in-hospital complication rates. Significant comorbidities included deficiency anemias, diabetes status, complicated hypertension, fluid and electrolyte disorders, neurological disorders, obesity, renal failure, weight loss, and osteoporosis (Table 2). 
Table 1. ICD-10 codes used to define vertebral compression fracture, operative treatment, and exclusion criteria

\begin{tabular}{|c|c|}
\hline Diagnosis & ICD-10 codes \\
\hline Osteoporotic vertebral compression fracture & M80.08XA, M80.88XA \\
\hline Operative treatment & $\begin{array}{l}\text { OPU43JZ, 0PU44JZ, 00U03JZ, 0QU04JZ, 0PS43ZZ, 0PU43JZ, 00S03ZZ, 00U03JZ, 0PU43JZ, 0PU44JZ, 0QU03JZ, } \\
\text { 00U04JZ }\end{array}$ \\
\hline \multicolumn{2}{|l|}{ Exclusion criteria } \\
\hline Fusion procedures & $\begin{array}{l}\text { OSG3070, OSG3071, OSG307J, OSG30A0, OSG30AJ, OSG30J0, OSG30J1, OSG30JJ, OSG30K0, OSG30K1, 0SG30KJ, } \\
\text { OSG3370, OSG3371, OSG337J, OSG33A0, OSG33AJ, OSG33J0, OSG33J1, OSG33JJ, 0SG33K0, 0SG33K1, } \\
\text { OSG33KJ, OSG3470, OSG3471, OSG347J, OSG34A0, OSG34AJ, 0SG34J0, 0SG34J1, 0SG34JJ, 0SG34K0, } \\
\text { OSG34K1, OSG34KJ }\end{array}$ \\
\hline Spinal decompression procedures & 00NX0ZZ, 00NX3ZZ, 00NX4ZZ, 00NY0ZZ, 00NY3ZZ, 00NY4ZZ \\
\hline Neoplasm & $\begin{array}{l}\text { C412, M8450XA, M8450XD, M8450XG, M8450XK, M8450XP, M8450XS, M8458XA, M8458XD, M8458XG, } \\
\text { M8458XK, M8458XP, M8458XS, M8460XA, M8460XD, M8460XG, M8460XK, M8460XP, M8460XS, M8468XA, } \\
\text { M8468XD, M8468XG, M8468XK, M8468XP, M8468XS, C419, C720, C729, C7940, C7949, C7951, C7952, C7989, } \\
\text { C799, C800, C801, C802, C768, D166, D168, D169, D334, D334, D321, D337, D339, D329, D367, D369, D480, } \\
\text { D487, D489, D492, D497, D4989, D499 }\end{array}$ \\
\hline Infection & $\begin{array}{l}\text { M4620, M4621, M4622, M4623, M4624, M4625, M4526, M4627, M4628, M4630, M4631, M4632, M4633, M4634, } \\
\text { M4635, M4536, M4637, M4638, M4639, M4640, M4641, M4642, M4643, M4644, M4645, M4546, M4647, } \\
\text { M4648, M4649, M4650, M4651, M4652, M4653, M4654, M4655, M4556, M4657, M4658, M4659, G061, G062, } \\
\text { G07, A1781 }\end{array}$ \\
\hline
\end{tabular}

ICD-10, International Classification of Diseases, 10th version.

Significant demographic features included sex, race, inhospital death, patient location, location/teaching status of hospital, hospital bed capacity, and hospital region (Table 3). All statistical tests were performed using Stata ver. 13.0 (Stata Corp., College Station, TX, USA), and two-sided $p$-values of less than were used to denote statistical significance.

\section{Results}

Cohort matching resulted in 14,850 patients who underwent operative treatment and 29,700 patients who underwent nonoperative treatment. The mean age of the patients in the operative (79.3\% female) and nonoperative (82.9\% female) treatment groups was 79.7 years and 79.6 years, respectively. All demographic and hospitalization characteristics are comprehensively outlined in Table 3.

On univariate analysis, the operative group demonstrated significantly lower rates of respiratory complications, such as pulmonary embolism (PE) (odds ratio [OR], 0.68 ; 95\% confidence interval [CI], 0.56-0.83; $p<0.001$ ), pneumonia (OR, $0.63 ; 95 \% \mathrm{CI}, 0.59-0.68 ; p<0.001$ ), and acute respiratory failure (OR, $0.84 ; 95 \% \mathrm{CI}, 0.78-0.91$; $p<0.001)$. Operative treatment was also associated with a significantly lower risk of cardiac complications, including myocardial infarction (MI) (OR, 0.34; 95\% CI, 0.28-0.42; $p<0.001$ ), cardiac arrest (OR, 0.52; 95\% CI, 0.35-0.78; $p=0.001$ ), acute heart failure (OR, $0.82 ; 95 \% \mathrm{CI}, 0.75-0.89$; $p<0.001$ ), and cardiogenic shock (OR, 0.40; 95\% CI, $0.23-$ $0.70 ; p<0.001)$; moreover, lower rates of cerebral infarction (OR, 0.61; 95\% CI, 0.49-0.77; $p<0.001$ ) were observed among patients undergoing operative treatment. The rates of infectious sequelae, including sepsis (OR, 0.42; 95\% CI, 0.37-0.46; $p<0.001)$ and septic shock (OR, 0.58; 95\% CI, $0.46-0.73 ; p<0.001$ ), were also significantly lower in the operative treatment group. Moreover, the occurrence of pressure ulcers (OR, 0.69; 95\% CI, 0.61-0.78; $p<0.001)$ and urinary tract infections (UTI) (OR, 0.92; 95\% CI, $0.88-0.97 ; p=0.001)$ was significantly decreased in the operative group compared with that in the nonoperative group. Conversely, patients undergoing operative treatment were more likely to experience acute renal failure (OR, 1.10; 95\% CI, 1.04-1.16; $p=0.001$ ) and radiculopathy (OR, 1.25; 95\% CI, 1.09-1.43; $p=0.002$ ) than those undergoing nonoperative treatment.

On multivariate analysis, operative treatment continued to demonstrate a significantly decreased risk of complications as previously observed. Significantly lower rates of pneumonia (OR, 0.75; 95\% CI, 0.67-0.84; $p<0.001)$ and acute respiratory failure (OR, $0.84 ; 95 \% \mathrm{CI}, 0.74-0.96$; $p=0.009$ ) persisted in the operative group. The risk of cardiac complications, including MI (OR, $0.20 ; 95 \% \mathrm{CI}$, $0.15-0.26 ; p<0.001$ ), acute heart failure (OR, $0.80 ; 95 \%$ CI, 0.69-0.91; $p=0.001$ ), and cardiogenic shock (OR, 0.23 ; 
Table 2. Prevalence of preexisting comorbidities at admission for operative and nonoperative treatment groups

\begin{tabular}{|c|c|c|c|}
\hline Comorbidities & Operative treatment & Nonoperative treatment & $p$-value \\
\hline Presence of at least one comorbidity & $14,525(97.8)$ & $29,050(97.8)$ & 1 \\
\hline Deficiency anemias & $795(5.4)$ & $1,955(6.6)$ & $0.023^{\mathrm{a}}$ \\
\hline Chronic blood loss anemia & $130(0.9)$ & $265(0.9)$ & 0.936 \\
\hline Alcohol abuse & $340(2.3)$ & $765(2.6)$ & 0.413 \\
\hline Rheumatoid arthritis/collagen vascular disease & $1,385(9.3)$ & $2,850(9.6)$ & 0.683 \\
\hline Congestive heart failure & $3,615(24.3)$ & $7,200(24.2)$ & 0.917 \\
\hline Chronic pulmonary disease & $5,400(36.4)$ & $10,575(35.6)$ & 0.482 \\
\hline Coagulopathy & $965(6.5)$ & $2,120(7.1)$ & 0.262 \\
\hline Depression & $2,785(18.8)$ & $5,405(18.2)$ & 0.523 \\
\hline Diabetes (uncomplicated) & $1,745(11.8)$ & $2,575(8.7)$ & $<0.001^{\text {a) }}$ \\
\hline Diabetes with chronic complications & $1,760(11.9)$ & $3,140(10.6)$ & 0.069 \\
\hline Drug abuse & $360(2.4)$ & $780(2.6)$ & 0.569 \\
\hline Hypertension (uncomplicated) & $7,570(51.0)$ & $14,315(48.2)$ & 0.013 \\
\hline Hypertension (complicated) & $4,070(27.4)$ & $7,410(25.0)$ & $0.012^{\mathrm{a}}$ \\
\hline Hypothyroidism & $3,730(25.1)$ & $7,200(24.2)$ & 0.365 \\
\hline Liver disease & $615(4.1)$ & $1,350(4.6)$ & 0.381 \\
\hline Lymphoma & $320(2.2)$ & $610(2.1)$ & 0.753 \\
\hline Fluid \& electrolyte disorders & $5,225(35.2)$ & $11,875(40.0)$ & $<0.001^{\text {a) }}$ \\
\hline Metastatic cancer & $175(1.2)$ & $475(1.6)$ & 0.118 \\
\hline Other neurological disorders & $1,620(10.9)$ & $3,920(13.2)$ & $0.002^{\mathrm{a}}$ \\
\hline Obesity & $1,260(8.5)$ & $1,940(6.5)$ & $0.001^{\mathrm{a}}$ \\
\hline Paralysis & $130(0.88)$ & $380(1.3)$ & 0.091 \\
\hline Peripheral vascular disorders & $1,555(10.5)$ & $3,370(11.4)$ & 0.214 \\
\hline Psychoses & $85(0.57)$ & $265(0.89)$ & 0.107 \\
\hline Pulmonary circulation disorders & $1,085(7.3)$ & $2,430(8.2)$ & 0.148 \\
\hline Renal failure & $2,945(19.8)$ & $5,195(17.5)$ & $0.007^{\mathrm{a}}$ \\
\hline Solid tumor without metastasis & $595(4.0)$ & $1,355(4.6)$ & 0.227 \\
\hline Peptic ulcer disease (without bleeding) & $160(1.1)$ & $305(1.03)$ & 0.825 \\
\hline Valvular disease & $1,760(11.9)$ & $3,270(11.0)$ & 0.237 \\
\hline Weight loss & $1,710(11.5)$ & $4,460(15.0)$ & $<0.001^{\text {a) }}$ \\
\hline Acquired immunodeficiency syndrome & $5(0.03)$ & $35(0.12)$ & 0.211 \\
\hline Osteoporosis & $450(3.0)$ & $1,220(4.1)$ & $0.012^{\mathrm{a}}$ \\
\hline Smoking & $1,400(9.4)$ & $2,855(9.6)$ & 0.779 \\
\hline
\end{tabular}

Values are presented as number (\%). All variables with corresponding $p$-values $<0.05$ were analyzed as independent variables on multivariate analysis.

alDenotes significance.

95\% CI, 0.10-0.53; $p=0.001)$ remained significantly lower in the operative group, as did the rate of cerebral infarction (OR, 0.44; 95\% CI, 0.32-0.62; $p<0.001$ ). Operative treatment continued to demonstrate a significantly decreased risk of infectious sequelae, specifically sepsis (OR, 0.39; 95\% CI, 0.34-0.45; $p<0.001)$ and septic shock (OR,
0.50; 95\% CI, 0.37-0.67; $p<0.001)$, compared with nonoperative treatment. The incidence of pressure ulcers (OR, $0.71 ; 95 \%$ CI, $0.60-0.85 ; p<0.001)$ remained significantly lower in the operative group as well. The only complication that had a higher rate in the operative group was acute renal failure (OR, 1.19; 95\% CI, 1.08-1.31; $p<0.001$ ). 
Table 3. Mean demographic characteristics of operative and nonoperative treatment groups

\begin{tabular}{|c|c|c|c|}
\hline Characteristic & Operative treatment $(n=14,850)$ & Nonoperative treatment $(n=29,700)$ & $p$-value \\
\hline Age (yr) & 79.69 & 79.62 & 0.745 \\
\hline Length of stay (day) & 6.73 & 5.54 & $<0.001^{\text {a }}$ \\
\hline No. of diagnoses & 16.32 & 16.78 & $<0.001^{\mathrm{a}}$ \\
\hline No. of procedures & 2.97 & 0.78 & $<0.001^{\text {al }}$ \\
\hline No. of ICD-10-CDM External Cause of Morbidity & 0.47 & 0.53 & 0.296 \\
\hline No. of days from admission to procedure & 3.34 & 2.31 & $<0.001^{\mathrm{a}}$ \\
\hline Total charge (USD) & 76,030 & 46,257 & $<0.001^{\mathrm{a}}$ \\
\hline Sex (female) & $11,780(79.3)$ & $24,630(82.9)$ & $<0.001^{a}$ \\
\hline Race & & & $0.001^{\mathrm{a}}$ \\
\hline White & $12,785(88.3)$ & $24,305(84.9)$ & \\
\hline Black & $280(1.9)$ & $825(2.9)$ & \\
\hline Hispanic & $750(5.2)$ & $1,730(6.04)$ & \\
\hline Asian or Pacific Islander & $395(2.7)$ & $1,100(3.8)$ & \\
\hline Native American & $50(0.35)$ & $100(0.35)$ & \\
\hline Other & $215(1.5)$ & $570(2.0)$ & \\
\hline Weekend admission & $3,595(24.2)$ & $7,460(25.9)$ & 0.076 \\
\hline Discharge Disposition ${ }^{\mathrm{bl}}$ & & & $<0.001^{a}$ \\
\hline Routine & $2,990(20.2)$ & $6,450(21.7)$ & \\
\hline Transfer to short-term hospital & $100(0.67)$ & $605(2.04)$ & \\
\hline Other transfers (nursing, intermediate, etc.) & $8,180(55.1)$ & $15,570(52.5)$ & \\
\hline Home health care & $3,455(23.3)$ & $6,340(21.4)$ & \\
\hline Against medical advice & $10(0.07)$ & $120(0.4)$ & \\
\hline In-hospital death & $100(0.67)$ & $600(2.0)$ & \\
\hline Transfer in status ${ }^{\text {b) }}$ & & & $0.011^{\mathrm{a}}$ \\
\hline Not transferred in & $13,500(91.4)$ & $26,510(89.6)$ & \\
\hline Transferred in from different acute care hospital & $715(4.8)$ & $1,565(5.3)$ & \\
\hline Transferred in from another type of health facility & $555(3.8)$ & $1,505(5.1)$ & \\
\hline Transfer out status ${ }^{\text {b) }}$ & & & $<0.001^{\mathrm{a}}$ \\
\hline Not transferred out & $6,555(44.2)$ & $13,510(45.5)$ & \\
\hline Transferred out to a different acute care hospital & $100(0.67)$ & $605(2.0)$ & \\
\hline Transferred out to another type of health facility & $8,180(55.1)$ & $15,570(52.5)$ & \\
\hline Primary payment method & & & 0.051 \\
\hline Medicare & $13,315(89.7)$ & $26,375(88.9)$ & \\
\hline Medicaid & $320(2.2)$ & $910(3.1)$ & \\
\hline Private insurance & $995(6.7)$ & $1,935(6.7)$ & \\
\hline Self-pay & $80(0.54)$ & $165(0.56)$ & \\
\hline No charge & 0 & $35(0.12)$ & \\
\hline Other payment & $140(0.94)$ & $210(0.71)$ & \\
\hline Patient location & & & $<0.001^{\mathrm{a}}$ \\
\hline Central counties of metro areas $>1$ million & $3,775(25.4)$ & $8,475(28.6)$ & \\
\hline Fringe counties of metro areas $>1$ million & $4,305(29.0)$ & $7,210(24.3)$ & \\
\hline
\end{tabular}


Table 3. Continued

\begin{tabular}{|c|c|c|c|}
\hline Characteristic & Operative treatment $(n=14,850)$ & Nonoperative treatment $(n=29,700)$ & $p$-value \\
\hline Counties in metro areas $250,000-1,000,000$ & $3,135(21.13)$ & $6,130(20.7)$ & \\
\hline Counties in metro areas $50,000-250,000$ & $1,700(11.5)$ & $3,045(10.3)$ & \\
\hline Micropolitan counties & $1,085(7.3)$ & $2,805(9.5)$ & \\
\hline Not metropolitan or micropolitan & $840(5.7)$ & $2,000(6.7)$ & \\
\hline Median household income & & & 0.678 \\
\hline 0-25th percentile & $3,065(20.9)$ & $6,030(20.6)$ & \\
\hline 26-50th percentile & $4,025(27.4)$ & $7,780(26.6)$ & \\
\hline 51-75th percentile & $4,040(27.5)$ & $8,100(27.6)$ & \\
\hline 76-100th percentile & $3,550(24.2)$ & $7,395(25.2)$ & \\
\hline Location/teaching status of hospital & & & $<0.001^{\text {al }}$ \\
\hline Rural & $895(6.03)$ & $3,190(10.7)$ & \\
\hline Urban, non-teaching & $3,630(24.4)$ & $6,890(23.2)$ & \\
\hline Urban, teaching & $10,325(69.5)$ & $19,620(66.1)$ & \\
\hline Hospital bed size capacity & & & $<0.001^{\mathrm{a}}$ \\
\hline Small & $2,460(16.6)$ & $6,460(21.8)$ & \\
\hline Medium & $4,805(32.4)$ & $8,510(28.7)$ & \\
\hline Large & $7,585(51.1)$ & $14,730(49.6)$ & \\
\hline Hospital region & & & $<0.001^{a \mid}$ \\
\hline Northeast & $2,305(15.5)$ & $6,540(22.02)$ & \\
\hline Midwest & $5,595(37.7)$ & $7,730(26.03)$ & \\
\hline South & $5,295(35.7)$ & $8,135(27.4)$ & \\
\hline West & $1,655(11.1)$ & $7,295(24.6)$ & \\
\hline
\end{tabular}

Values are presented as number or number (\%).

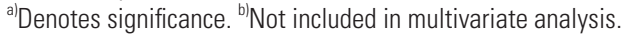

No significant differences in the incidence of deep vein thrombosis, PE, cardiac arrest, UTI, and radiculopathy were observed between the two groups. All complication rates and corresponding significance values are listed in Table 4.

Operative treatment was associated with a higher mean total cost of admission and mean LOS at $\$ 76,030$ and 6.73 days, respectively, compared with nonoperative treatment at $\$ 46,257$ and 5.54 days, respectively (both $p<0.001$ ). Among patients who underwent operative treatment, $69.5 \%$ were admitted to a metropolitan teaching hospital, $24.4 \%$ were admitted to a metropolitan nonteaching hospital, and $6.03 \%$ were admitted to a rural hospital compared with $66.1 \%, 23.2 \%$, and $10.7 \%$, respectively, in the nonoperative group $(p<0.001)$. Overall, nonoperative treatment was associated with a significantly greater inhospital mortality rate at $2 \%$ than operative treatment at $0.67 \%(p<0.001)$ (Table 3$)$.

\section{Discussion}

In this study, we found that patients hospitalized for OCFs who undergo conservative treatment have an increased rate of acute in-hospital complications, including respiratory complications, cardiac complications, sepsis, and pressure ulcerations, compared with those who undergo acute PVA within 1 week. After PVA, patients are encouraged to mobilize and have been shown to have decreased pain $[4,14,15]$. Mobilization significantly alters the morbidity and mortality rates $[16,17]$. This may explain the increased rate of acute complications seen in patients who underwent conservative treatment in this study. Contrarily, patients who undergo PVA have a higher rate of acute renal failure. Acute renal failure is a known postoperative complication, explaining the increased risk in patients treated operatively [18-21].

Our findings are supported by several studies that exam- 
Table 4. Comparison of complication rates between operative and nonoperative treatment groups using univariate and multivariate analysis with corresponding odds ratios and $p$-values

\begin{tabular}{|c|c|c|c|c|c|c|}
\hline \multirow{2}{*}{ Complications } & \multirow{2}{*}{ Operative treatment } & \multirow{2}{*}{ Nonoperative treatment } & \multicolumn{2}{|c|}{ Univariate analysis } & \multicolumn{2}{|c|}{ Multivariate analysis } \\
\hline & & & OR $(95 \% \mathrm{CI})$ & $p$-value & $\mathrm{OR}(95 \% \mathrm{Cl})$ & $p$-value \\
\hline Deep vein thrombosis & $160(1.1)$ & $380(1.3)$ & $0.84(0.70-1.01)$ & 0.067 & $1.11(0.85-1.47)$ & 0.446 \\
\hline Pulmonary embolism & $135(0.91)$ & $395(1.3)$ & $0.68(0.56-0.83)$ & $<0.001^{a)}$ & $0.77(0.57-1.04)$ & 0.089 \\
\hline Cerebral infarct & $100(0.67)$ & $325(1.1)$ & $0.61(0.49-0.77)$ & $<0.001^{a)}$ & $0.44(0.32-0.62)$ & $<0.001^{\text {a) }}$ \\
\hline Pneumonia & $1,100(7.41)$ & $3,345(11.3)$ & $0.63(0.59-0.68)$ & $<0.001^{a)}$ & $0.75(0.67-0.84)$ & $<0.001^{\text {a) }}$ \\
\hline Acute respiratory failure & $1,120(7.5)$ & $2,625(8.8)$ & $0.84(0.78-0.91)$ & $<0.001^{a)}$ & $0.84(0.74-0.96)$ & $0.009^{a l}$ \\
\hline Myocardial infarction & $110(0.74)$ & $640(2.2)$ & $0.34(0.28-0.42)$ & $<0.001^{a)}$ & $0.20(0.15-0.26)$ & $<0.001^{\text {a) }}$ \\
\hline Cardiac arrest & $30(0.2)$ & $115(0.39)$ & $0.52(0.35-0.78)$ & $0.001^{\text {a) }}$ & $0.58(0.33-1.03)$ & 0.061 \\
\hline Acute heart failure & $805(5.4)$ & $1,940(6.5)$ & $0.82(0.75-0.89)$ & $<0.001^{\text {a) }}$ & $0.80(0.69-0.91)$ & $0.001^{\text {a) }}$ \\
\hline Cardiogenic shock & $15(0.1)$ & $75(0.25)$ & $0.40(0.23-0.70)$ & $0.001^{\text {a) }}$ & $0.23(0.10-0.53)$ & $0.001^{\text {al }}$ \\
\hline Acute renal failure & $2,265(15.3)$ & $4,175(14.6)$ & $1.10(1.04-1.16)$ & $0.001^{\text {a) }}$ & $1.19(1.08-1.31)$ & $<0.001^{\text {a) }}$ \\
\hline Urinary tract infection & $2,700(18.2)$ & $5,775(19.4)$ & $0.92(0.88-0.97)$ & $0.001^{\text {a) }}$ & $1.02(0.94-1.11)$ & 0.581 \\
\hline Sepsis & $430(2.9)$ & $1,990(6.7)$ & $0.42(0.37-0.46)$ & $<0.001^{a \mid}$ & $0.39(0.34-0.45)$ & $<0.001^{\text {a) }}$ \\
\hline Septic shock & $95(0.64)$ & 325 (1.09) & $0.58(0.46-0.73)$ & $<0.001^{\text {a) }}$ & $0.50(0.37-0.67)$ & $<0.001^{\text {a) }}$ \\
\hline Pressure ulcer & $355(2.4)$ & $1,020(3.4)$ & $0.69(0.61-0.78)$ & $<0.001^{a)}$ & $0.71(0.60-0.85)$ & $<0.001^{\text {a) }}$ \\
\hline Radiculopathy & $335(2.3)$ & $540(1.8)$ & $1.25(1.09-1.43)$ & $0.002^{\mathrm{al}}$ & $1.10(0.89-1.36)$ & 0.363 \\
\hline
\end{tabular}

Values are presented as number (\%), unless otherwise stated.

$\mathrm{OR}$, odds ratio; $\mathrm{Cl}$, confidence interval.

a) Denotes significance.

ined the morbidity and mortality benefits of kyphoplasty and vertebroplasty. Eddin et al. [12] have found that both kyphoplasty and vertebroplasty offer improved mortality benefits compared with nonoperative treatment at up to 4 years following OCF in a national database study. A metaanalysis by Yuan et al. [22] has found that vertebroplasty and kyphoplasty improve quality of life, pain, and functional outcomes. Yang et al. [8] have reported that early vertebroplasty in the aged population offered faster and better pain relief and improved functional outcomes with lower complication rates than conservative therapy. Similarly, Lin et al. [9] have found improved morbidity and mortality with acute versus subacute vertebroplasty in the aged population. Clark et al. [14] have recently published a randomized controlled trial, which showed that vertebroplasty is superior to sham vertebroplasty in reducing pain, improving functional outcome scores, and decreasing analgesic use up to 6 months following OCF.

In a prospective study, Hoshino et al. have found that patients treated with kyphoplasty within 2 months of a painful OCF were less likely to have a decrease in activities of daily living [11]. Similarly, Wardlaw et al. [10] have conducted a randomized clinical trial and found that kyphoplasty resulted in significantly greater improvements in quality of life and disability measures and reduction in back pain than nonoperative treatment in patients with acute painful vertebral fractures. These differences, however, diminished at 12 months. Our findings add to this body of literature, which indicates an improved morbidity benefit with PVA compared with nonoperative management.

Conversely, several publications refute these beneficial outcomes. In a randomized controlled trial, Firanescu et al. [6] have found that vertebroplasty offers no improvements in pain and functional outcomes compared with a sham procedure. Kallmes et al. [7] had similar findings in a separate randomized controlled trial with analogous methods. However, these studies assessed pain and functional outcomes and were not powered to detect differences in morbidity between the two treatment groups.

This study has several limitations. It is a national database study, so it lacks granularity on the patient level. Because of this, we could not report on fracture characteristics or radiographic parameters. An inherent selection 
bias exists when choosing patients for operative versus nonoperative management. A subset of patients selected for nonoperative management are likely too sick for operative treatment, despite having operative indications. While we matched our cohorts based on several comorbidities and controlled for many potential confounding factors, it is possible that we did not exclude this subset of patients. Additionally, although we believe that early mobilization may contribute to the improved morbidity benefit of acute PVA, we did not have data that support this theory because it is not recorded in the NIS database. Finally, the NIS database is an inpatient-only database, so we could not capture outpatient procedures or long-term complications following initial hospitalization.

\section{Conclusions}

Patients who undergo acute PVA for OCF have lower rates of respiratory complications, cardiac complications, sepsis, and pressure ulcerations, while having a higher rate of acute renal failure. This adds to the body of literature that supports that PVA offers morbidity and mortality benefits compared with nonoperative management.

\section{Conflict of Interest}

No potential conflict of interest relevant to this article was reported.

\section{References}

1. Burge R, Dawson-Hughes B, Solomon DH, Wong JB, King A, Tosteson A. Incidence and economic burden of osteoporosis-related fractures in the United States, 2005-2025. J Bone Miner Res 2007;22:465-75.

2. Hansen D, Bazell C, Pelizzari P, Pyenson B. Medicare cost of osteoporotic fractures: the clinical and cost burden of an important consequence of osteoporosis [Internet]. Seattle (WA): Milliman; 2019 [cited 2020 Oct 10]. Available from: https://www.milliman.com/en/insight/medicare-cost-ofosteoporotic-fractures.

3. Goldstein CL, Chutkan NB, Choma TJ, Orr RD. Management of the elderly with vertebral compression fractures. Neurosurgery 2015;77 Suppl 4:S33-45.

4. Rousing R, Andersen MO, Jespersen SM, Thomsen K, Lauritsen J. Percutaneous vertebroplasty compared to conservative treatment in patients with painful acute or subacute osteoporotic vertebral fractures: threemonths follow-up in a clinical randomized study. Spine (Phila Pa 1976) 2009;34:1349-54.

5. Buchbinder R, Johnston RV, Rischin KJ, et al. Percutaneous vertebroplasty for osteoporotic vertebral compression fracture. Cochrane Database Syst Rev 2018;4:CD006349.

6. Firanescu CE, de Vries J, Lodder P, et al. Vertebroplasty versus sham procedure for painful acute osteoporotic vertebral compression fractures (VERTOS IV): randomised sham controlled clinical trial. BMJ 2018;361:k1551.

7. Kallmes DF, Comstock BA, Heagerty PJ, et al. A randomized trial of vertebroplasty for osteoporotic spinal fractures. N Engl J Med 2009;361:569-79.

8. Yang EZ, Xu JG, Huang GZ, et al. Percutaneous vertebroplasty versus conservative treatment in aged patients with acute osteoporotic vertebral compression fractures: a prospective randomized controlled clinical study. Spine (Phila Pa 1976) 2016;41:653-60.

9. Lin JH, Chien LN, Tsai WL, Chen LY, Chiang YH, Hsieh YC. Early vertebroplasty associated with a lower risk of mortality and respiratory failure in aged patients with painful vertebral compression fractures: a population-based cohort study in Taiwan. Spine J 2017;17:1310-8.

10. Wardlaw D, Cummings SR, Van Meirhaeghe J, et al. Efficacy and safety of balloon kyphoplasty compared with non-surgical care for vertebral compression fracture (FREE): a randomised controlled trial. Lancet 2009;373:1016-24.

11. Hoshino M, Takahashi S, Yasuda H, et al. Balloon kyphoplasty versus conservative treatment for acute osteoporotic vertebral fractures with poor prognostic factors: propensity score matched analysis using data from two prospective multicenter studies. Spine (Phila Pa 1976) 2019;44:110-7.

12. Edidin AA, Ong KL, Lau E, Kurtz SM. Mortality risk for operated and nonoperated vertebral fracture patients in the medicare population. J Bone Miner Res 2011;26:1617-26.

13. McGuire R. AAOS clinical practice guideline: the treatment of symptomatic osteoporotic spinal compression fractures. J Am Acad Orthop Surg 2011;19:183-4.

14. Clark W, Bird P, Gonski P, et al. Safety and efficacy of vertebroplasty for acute painful osteoporotic frac- 
tures (VAPOUR): a multicentre, randomised, doubleblind, placebo-controlled trial. Lancet 2016;388:140816.

15. Voormolen MH, Mali WP, Lohle PN, et al. Percutaneous vertebroplasty compared with optimal pain medication treatment: short-term clinical outcome of patients with subacute or chronic painful osteoporotic vertebral compression fractures: the VERTOS study. AJNR Am J Neuroradiol 2007;28:555-60.

16. Brown CJ, Friedkin RJ, Inouye SK. Prevalence and outcomes of low mobility in hospitalized older patients. J Am Geriatr Soc 2004;52:1263-70.

17. Siu AL, Penrod JD, Boockvar KS, Koval K, Strauss E, Morrison RS. Early ambulation after hip fracture: effects on function and mortality. Arch Intern Med 2006;166:766-71.

18. Mavrogenis A, Mitsiokapa EA, Romantini M, et al. Acute renal failure in orthopaedic surgery. J Long Term Eff Med Implants 2011;21:149-58.
19. Pedersen AB, Christiansen CF, Gammelager H, Kahlert J, Sorensen HT. Risk of acute renal failure and mortality after surgery for a fracture of the hip: a population-based cohort study. Bone Joint J 2016;98B:1112-8.

20. Ostermann M, Cennamo A, Meersch M, Kunst G. A narrative review of the impact of surgery and anaesthesia on acute kidney injury. Anaesthesia 2020;75 Suppl 1:e121-33.

21. Romagnoli S, Ricci Z, Ronco C. Perioperative acute kidney injury: prevention, early recognition, and supportive measures. Nephron 2018;140:105-10.

22. Yuan WH, Hsu HC, Lai KL. Vertebroplasty and balloon kyphoplasty versus conservative treatment for osteoporotic vertebral compression fractures: a metaanalysis. Medicine (Baltimore) 2016;95:e4491. 